04

\title{
Регистрация ультрафиолетового излучения широких атмосферных ливней: перспективы для черенковской гамма-астрономии
}

\author{
() Е.Е. Холупенко, ${ }^{1}$ А.М. Быков, ${ }^{1}$ Ф.А. Агаронян, ${ }^{2,3}$ Г.И. Васильев, ${ }^{1}$ А.М. Красильщиков, ${ }^{1}$ П.Н. Аруев, ${ }^{1}$ \\ В.В. Забродский, ${ }^{1}$ А.В. Николаев ${ }^{1}$ \\ ${ }^{1}$ Физико-технический институт им. А.Ф. Иоффре РАН, \\ 194021 Санкт-Петербург, Россия \\ ${ }^{2}$ Dublin Institute of Advanced Studies \\ ${ }^{3}$ Max-Planck-Institut für Kernphysik \\ e-mail: eugene@astro.ioffe.ru
}

(Поступило в Редакцию 2 мая 2018 г.)

Представлены результаты моделирования черенковского излучения широких атмосферных ливней (ШАЛ), порожденных космическими гамма-квантами и протонами космических лучей. Для наблюдений на высоте 2 и $5 \mathrm{~km}$ над уровнем моря рассчитана спектральная плотность черенковского излучения ШАЛ в диапазоне 240-700 nm при энергиях первичных частиц $3 \mathrm{GeV}-10 \mathrm{TeV}$. Отношение количества фотонов черенковского излучения ШАЛ в оптическом и ультрафиолетовом (УФ) диапазоне существенно зависит от типа первичной частицы, поэтому измерение и анализ этого отношения может применяться для выделения гаммасобытий из фона, создаваемого частицами космических лучей. Использование детектирующих элементов на основе специализированных кремниевых фотоумножителей, обладающих высокой чувствительностью в УФ диапазоне, позволяет существенно увеличить продолжительность рабочего цикла черенковского гаммателескопа за счет наблюдений в лунные ночи. При использовании в телескопах черенковской гаммаобсерватории ALEGRO разработанных в ФТИ им. А.Ф. Иоффе кремниевых лавинных умножителей (SiPM), порог регистрации гамма-событий только на основе измерений в УФ диапазоне составит около $40 \mathrm{GeV}$ на высоте $5 \mathrm{~km}$ и около $80 \mathrm{GeV}$ на высоте $2 \mathrm{~km}$.

DOI: 10.21883/JTF.2018.11.46626.176-18

\section{1. Введение}

В последние десятилетия наблюдается значительный прогресс в создании основных инструментов наземной гамма-астрономии - черенковских гамма-телескопов, которые регистрируют излучение широких атмосферных ливней (ШАЛ), вызванных попаданием в атмосферу Земли космических $\gamma$-квантов. Происходит как количественное, так и качественное развитие таких инструментов: увеличение размеров зеркал (например, $28 \mathrm{~m}$ зеркало телескопа CT5 H.E.S.S. II по сравнению с $12 \mathrm{~m}$ зеркалами телескопов CT1-4 H.E.S.S. [1]), использование вспомогательных мюонных датчиков и датчиков радиоизлучения, а также использование полупроводниковых фотодетекторов (например, в камерах телескопа FACT [2]) вместо вакуумных фотоумножителей (ФЭУ).

Разработка черенковских гамма-обсерваторий нового поколения требует исследования ряда перспективных технологий, позволяющих повысить эффективность детектирования космических $\gamma$-квантов. Одной из таких возможностей является регистрация и анализ ультрафиолетового (УФ) излучения ШАЛ. Эта технология была впервые предложена и апробирована на черенковских телескопах второго поколения ГТ-48 [3,4] и Whipple $[5,6]$. Она позволяет добиться значительного увеличения длительности рабочего цикла [7], а при использовании совместно с регистрацией оптического черенковского излучения ШАЛ позволяет повысить эффективность идентификации типа первичной частицы, вызвавшей ШАЛ (так называемой гамма-адронной сепарации). Вопрос об эффективности гамма-адронной сепарации посредством измерения доли УФ излучения в черенковском излучении ШАЛ для высот наблюдения до $2 \mathrm{~km}$ был также исследован теоретически М.А. Рахманом и его коллегами $[8,9]$, но в настоящей работе для моделирования использованы более адекватные и взаимосогласованные зависимости плотности атмосферы и показателя преломления от высоты (см. подробнее разд. 3.2). Регистрация УФ излучения ШАЛ была недавно апробирована на телескопах третьего поколения VERITAS [10] (высота $\simeq 1.3 \mathrm{~km})$ и MAGIC $[11,12]$ (высота $\simeq 2.2 \mathrm{~km})$ для увеличения длительности рабочего цикла. Тем не менее важно отметить, что использование только УФ данных приводит к ухудшению некоторых параметров телескопа, в частности, к увеличению пороговой энергии регистрации $\gamma$-квантов. Насколько нам известно, на данном этапе развития проекта черенковской гаммаобсерватории СТА [13] регистрация УФ излучения ШАЛ телескопами этой обсерватории не планируется.

Для создания черенковских гамма-телескопов нового (четвертого) поколения необходимо реализовать ряд подходов, которые будут качественно отличать их от установок третьего поколения - H.E.S.S., MAGIC и VERITAS. Среди этих подходов можно выделить использование зеркал больших размеров (диаметром 20-30 m), использование кремниевых фотоумножителей (SiPM) 
вместо традиционных вакуумных ФЭУ, а также расположение обсерваторий на большой высоте над уровнем моря (a.s.1.) — вплоть до $5 \mathrm{~km}$ [14-16].

Расположение обсерватории на большой высоте и увеличенная собирающая поверхность зеркал вновь делают актуальным вопрос о целесообразности регистрации УФ излучения ШАЛ, которое можно использовать для увеличения надежности идентификации типа первичной частицы и увеличения длительности рабочего цикла. Настоящая работа посвящена исследованию этих вопросов и, в частности, оценке пороговой энергии наблюдений на перспективной гамма-обсерватории ALEGRO [16] при использовании камер на основе разработанных в ФТИ им. А.Ф. Иоффе детекторов SiPM с чувствительностью в далеком УФ диапазоне [17], а также с учетом большого диаметра зеркал $(30 \mathrm{~m})$ и планируемой высоты расположения $\simeq 5 \mathrm{~km}$.

Расчеты проведены с помощью полуаналитической модели, в которой эволюция источников черенковского излучения (высокоэнергетических вторичных электронов и позитронов ШАЛ) описывалась с помощью аналитических приближений для средних продольных профилей ШАЛ от $\gamma$-квантов (приближение Грейзена [18]) и от протонов космических лучей (приближение Грейзена-Ильиной-Линсли [19]), а распространение черенковского излучения ШАЛ описывалось с помощью уравнения переноса, которое решалось численно.

\section{2. Модель распространения ШАЛ и генерации черенковского излучения}

\section{1. Формирование и распространение черенковского излучения ШАЛ}

Формирование и распространение черенковского излучения ШАЛ может быть описано редуцированным уравнением переноса излучения:

$$
-\frac{N_{\mathrm{Ch}}}{\partial h}=Q-\mu N_{\mathrm{Ch}}, \quad N_{\mathrm{Ch}}\left(h_{\max }, \lambda\right)=0,
$$

где $N_{\mathrm{Ch}}(h, \lambda)\left[\mathrm{cm}^{-1}\right]$ - спектральная плотность (количество черенковских фотонов в пучке ШАЛ в единичном интервале длин волн) на высоте $h$ над уровнем моря на длине волны $\lambda, Q(h, \lambda)\left[\mathrm{cm}^{-2}\right]$ - количество черенковских фотонов, порожденных вторичными электронами и позитронами ШАЛ ${ }^{1}$ в единичном интервале длин волн на единичном интервале пути, $\mu\left[\mathrm{cm}^{-1}\right]-$ суммарный эффективный коэффициент поглощения (подробнее см. разд. 2.3), $h_{\max }$ - условная высота верхней границы атмосферы (в настоящей работе принято значение $46.7 \mathrm{~km})$.

\footnotetext{
1 Далее в тексте под термином „электроны ШАЛ“ будут пониматься вторичные электроны и позитроны ШАЛ. Недостаток количества позитронов вследствие аннигиляции (см., например, $[20,21])$ в настоящей работе не учитывается.
}

Редуцированное уравнение переноса (1) может быть получено из стандартной формы уравнения переноса для интенсивности излучения $I_{\lambda}\left[\mathrm{cm}^{-3} \mathrm{~s}^{-1} \mathrm{ster}^{-1}\right]$ посредством интегрирования по времени (в пределах длительности черенковской вспышки от ШАЛ), поперечному сечению ШАЛ (в пределах нескольких мольеровских радиусов, $\left.\sim 4 r_{\mathrm{M}} \approx 250-300 \mathrm{~m}\right)$ и телесному углу в пределах совокупности конусов черенковского излучения электронов ШАЛ (что соответствует полному углу раствора $\lesssim 5^{\circ}$ ).

Величина $Q(h)$ может быть задана следующей формулой:

$$
Q(h)=\int_{E_{\mathrm{th}}}^{\infty} \frac{\partial N_{e}}{\partial E_{e}} q\left(h, E_{e}\right) d E_{e},
$$

где $\partial N_{e} / \partial E_{e}-$ функция распределения электронов ШАЛ по энергии (подробнее см. разд. 2.2.1), $E_{\mathrm{th}}-$ пороговая энергия электронов, при которой начинает генерироваться черенковское излучение, $q\left(h, E_{e}\right)-$ количество черенковских фотонов, сгенерированных одним электроном в единичном интервале длин волн на единичном интервале пути, определяемое по формуле Франка-Тамма:

$$
q\left(h, E_{e}\right)=\frac{2 \pi \alpha}{\lambda^{2}}\left(1-\beta^{-2} n^{-2}\right),
$$

где $\alpha$ - постоянная тонкой структуры, $\beta=v / c-$ скорость электрона в единицах скорости света, $n(h, \lambda)-$ показатель преломления для воздуха на высоте $h$ над уровнем моря, на длине волны $\lambda$ (подробнее см. разд. 2.3). Величина $\beta$ связана с энергией электрона соотношением

$$
\beta=\sqrt{1-\left(\frac{m_{e} c^{2}}{E_{e}}\right)^{2}},
$$

где $m_{e}-$ масса электрона. Пороговая энергия $E_{\mathrm{th}}$ в формуле (2) определяется из условия $q\left(h, E_{\mathrm{th}}\right)=0$ или $\beta\left(E_{\mathrm{th}}\right)=n^{-1}$.

\section{2. Электрон-фотонная компонента ШАЛ}

\subsection{1. Распределение электронов ШАЛ по энергии}

Функция распределения электронов ШАЛ по энергии может быть представлена в следующей форме:

$$
\frac{\partial N_{e}}{\partial E_{e}}=\frac{N_{e}(h)}{E_{c}} g_{e}(\epsilon),
$$

где $E_{c}-$ критическая энергия развития электронфотонной лавины в воздухе (см., например, [22]), $N_{e}-$ полное количество электронов ШАЛ на высоте $h$ (подробнее см. разд. 2.2.2 и 2.2.3). Следует отметить, что в отличие от теории переноса излучения, в которой основные величины (интенсивность, поток излучения) рассматриваются в зависимости от пройденного пути 
(в нашем случае, от высоты $h$ ), в теории лавин энергичных вторичных частиц в различных средах (твердых телах, жидкостях, газах) исследуемые величины рассматривают, как правило, в зависимости не от пройденного пути, а от глубины среды $x\left[\mathrm{~g} \cdot \mathrm{cm}^{-2}\right]$. В данном случае глубина атмосферы связана с высотой над уровнем моря следующей формулой:

$$
x(h)=\int_{h}^{\infty} \rho\left(h^{\prime}\right) d h^{\prime},
$$

где $\rho(h)$ - плотность воздуха на высоте $h$. Поэтому далее в тексте следующие обозначения будут считаться равнозначными: $F(h)=F(x)$, где $F-$ произвольная функция высоты (подробнее о связи глубины атмосферы и высоты см. разд. 2.3). Величина $g(\epsilon)$ - плотность вероятности, адаптированная по работе Гиллера и др. [22]:

$$
\begin{aligned}
g_{e}(\epsilon) & =A_{e}(s) \epsilon^{-1}\left[1-a_{e} \exp \left(-d_{e}(s) \epsilon\right)\right] \\
& \times(1+\epsilon)^{-\left[s+b_{e} \ln \left(\epsilon / c_{e}\right)\right]}
\end{aligned}
$$

где $\epsilon=E_{e} / E_{c}-$ энергия электронов ШАЛ в единицах критической энергии, $a_{e}=1.005, b_{e}=0.06, c_{e}=189$, $d_{e}(s)=7.06 \cdot s+12.48, A_{e}(s)=0.111 \cdot s+0.134$. Это приближение для функции распределения справедливо в интервале значений возраста ШАЛ $s=0.7-1.3$ (определение этой величины дано ниже в разделах 2.2.2 и 2.2.3). Плотность вероятности $g_{e}(\epsilon)$ нормирована так, что

$$
\int_{0}^{\infty} g_{e}(\epsilon) d \epsilon=1 \text {. }
$$

\subsection{2. ШАЛ, вызванные $\gamma$-квантами}

Для описания зависимости количества электронов ШАЛ, рожденных в атмосфере Земли космическими $\gamma$-квантами высоких энергий (,رШАЛ“), от глубины атмосферы было использованно приближение Грейзена $[18,8]$ :

$$
N_{e}^{\gamma}(x, E)=\frac{0.31}{\sqrt{t_{\gamma m}}} \exp \left(t_{\gamma}\left[1-1.5 \ln \left(s_{\gamma}\right)\right]\right),
$$

где $E-$ энергия первичной частицы, $t_{\gamma}=x / x_{r}$ - относительная глубина атмосферы (в единицах радиационной длины в воздухе $\left.x_{r}=37.15 \mathrm{~g} \cdot \mathrm{cm}^{-2}\right), t_{\gamma m}=x_{\gamma m} / x_{r}-$ относительная глубина максимума $\gamma$ ШАЛ, $s_{\gamma}$ - возраст $\gamma$ ШАЛ.

Позиция максимума $\gamma$ ШАЛ может быть определена как

$$
t_{\gamma m}=\ln \left(E / E_{\gamma c}\right),
$$

где $E_{\gamma c}=84.2 \mathrm{MeV}$ - критическая энергия электронов в воздухе (примерно равна энергии, при которой сравниваются потери на излучение и ионизацию, см., например, [8,18] и ссылки в этих работах). Возраст $\gamma$ ШАЛ определяется как

$$
s_{\gamma}=\frac{3 t_{\gamma}}{t_{\gamma}+2 t_{\gamma m}} .
$$

Количество заряженных частиц в лавине достигает максимального значения при $s_{\gamma}=1, t_{\gamma}=t_{\gamma m}$ и равняется

$$
N_{e, \max }^{\gamma}(E)=\frac{0.31 E}{E_{\gamma c} \sqrt{\ln \left(E / E_{\gamma c}\right)}} .
$$

\subsection{3. ШАЛ, вызванные протонами}

Для описания зависимости количества электронов ШАЛ, вызванных в атмосфере Земли космическими протонами высоких энергий („рШАЛ“), от глубины атмосферы было использовано приближение Грейзена-Ильиной-Линсли [19,23,24]:

$$
N_{e}^{p}(x, E)=\frac{E}{E_{p}} \exp \left(t_{p}-t_{p m}-2 t \ln \left(s_{p}\right)\right)
$$

где $E_{p}=1.45 \mathrm{GeV}-$ нормировочная энергия (индекс ,,$p^{\text {“ } о з н а ч а е т ~}$, опотон“" $), t_{p}=\left(x-x_{p 1}\right) / x_{r}$ - относительная глубина атмосферы, отсчитанная от глубины первого взаимодействия $x_{p 1}, t_{p m}$ - относительная глубина максимума рШАЛ, $s_{p}$ - возраст рШАЛ. Значение $t_{p m}$ дается следующей формулой (см., например, [24]):

$$
t_{p m}=a+b\left[\ln \left(E / E_{p c}\right)-\ln A\right]=a+b \ln \left(E / E_{p c}\right),
$$

где $a=1.7, b=0.76, A-$ атомная масса первичной частицы (для протона $A=1$ ). Значение критической энергии $E_{p c}$ в этом случае принято равным $81 \mathrm{MeV}$; его отличие от значения $E_{\gamma c}$ (см. разд. 2.2.2) обусловлено тем, что оно подобрано из условия наилучшего соответствия среднего продольного профиля ШАЛ (13) результатам численного моделирования методом Монте-Карло (см. [24] и ссылки в этой работе). Возраст рШАЛ $s_{p}$ дается следующим соотношением:

$$
s_{p}=\frac{2 t_{p}}{t_{p}+t_{p m}} .
$$

Среднее значение глубины первого взаимодействия может быть оценено по следующей формуле [25]:

$$
x_{p 1}=83.1(E[G e V])^{-0.052} \mathrm{~g} \cdot \mathrm{cm}^{-2} .
$$

Количество заряженных частиц в лавине достигает максимального значения при $s_{p}=1, t_{p}=t_{p m}$ и равняется

$$
N_{e, \max }^{p}(E)=\frac{E}{E_{p}} .
$$

\section{3. Модель атмосферы}

Модель атмосферы как среды, в которой происходит формирование ШАЛ, генерация и распространение черенковского излучения, должна включать описание 
таких ее свойств как плотность воздуха (или связь глубины атмосферы с высотой), преломление, поглощение и рассеяние излучения.

Связь глубины атмосферы и высоты (для высот $h \geq 2 \mathrm{~km}$ ) может быть описана следующей формулой:

$$
h=H_{0}+\left(H_{1}+H_{2} x\right) \ln \left(x_{0} / x\right),
$$

где $H_{0}=4 \cdot 10^{5} \mathrm{~cm}, H_{1}=6.62 \cdot 10^{5} \mathrm{~cm}, H_{2}=160 \mathrm{~cm}^{3} \cdot \mathrm{g}^{-1}$, $x_{0}=631 \mathrm{~g} \cdot \mathrm{cm}^{-2}$ - глубина атмосферы на высоте $4 \mathrm{~km}$ (согласно Хеку и др. [26], полная глубина атмосферы, соответствующая высоте $h=0 \mathrm{~cm}$ над уровнем моря, составляет $\simeq 1036 \mathrm{~g} \cdot \mathrm{cm}^{-2}$ ). Аппроксимация (18) в интервале высот $1.8 \mathrm{~km} \leq h \leq 36 \mathrm{~km}$ (что соответствует интервалу значений глубины атмосферы $\left.830 \mathrm{~g} \cdot \mathrm{cm}^{-2} \gtrsim x \gtrsim 5 \mathrm{~g} \cdot \mathrm{cm}^{-2}\right)$ согласуется с функцией $h(x)$, которая может быть получена из параметризации Линсли („Linsley Model of 5 layers“) с параметрами Хека [26] для функции $x(h)$ в рамках модели стандартной атмосферы [27], с относительной точностью порядка $1 \%$. Поскольку функция (18) гладкая, ее удобно использовать для нахождения зависимости плотности воздуха от глубины атмосферы в соответствии с формулой $\rho=-d x / d h$ (следует из определения глубины атмосферы (6)), что в явном виде дает

$$
\rho(x)=\frac{x}{H_{1}+H_{2} x\left(1-\ln x_{0} / x\right)} .
$$

Показатель преломления воздуха (атмосферы) дается выражением $n=1+\Delta n$, где $\Delta n(x, \lambda)$ - преломляющая способность (refractivity) воздуха. Зависимость преломляющей способности от глубины атмосферы (высоты) может быть определена на основании соотношения Гладстона-Дейла:

$$
\Delta n(x, \lambda)=\Delta n_{0}(\lambda) \frac{\rho(x)}{\rho_{0}},
$$

где $\Delta n_{0}(\lambda)$ - величина преломляющей способности воздуха на уровне моря на длине волны $\lambda$, $\rho_{0}=1.23 \cdot 10^{-3} \mathrm{~g} \cdot \mathrm{cm}^{-3}$ - плотность воздуха на уровне моря. В настоящей работе использовано приближение постоянной (не зависящей от длины волны) величины $\Delta n_{0}=2.8 \cdot 10^{-4}$, соответствующей преломляющей способности воздуха на длине волны $\lambda \simeq 450 \mathrm{~nm}$ [28], находящейся примерно посередине исследуемого диапазона 240-700 nm, а также соответствующей максимуму чувствительности стандартного SiPM Hamamatsu серии S10362-33 [29].

Следует отметить, что величина $\Delta n_{0}(\lambda)$ изменяется в указанном диапазоне от $3.04 \cdot 10^{-4}$ до $2.76 \cdot 10^{-4}$ (по данным [28]), то есть, она уменьшается с увеличением длины волны примерно на 10\%. Такое изменение показателя преломления приводит к сопоставимому $(\sim 0.1)$ изменению темпа генерации черенковского излучения (2). В то же время, основная зависимость темпа генерации $Q(h, \lambda)$ от длины волны описывается фактором $\lambda^{-2}$, что в указанном диапазоне длин волн составляет величину
$Q(h, 240 \mathrm{~nm}) / Q(h, 700 \mathrm{~nm}) \simeq 8.5$. Таким образом, зависимостью преломляющей способности $\Delta n_{0}$ от длины волны в рассматриваемой ситуации можно пренебречь. Кроме того, следует отметить, что точный учет зависимости $\Delta n_{0}$ от длины волны приводит к значительным вычислительным сложностям, поскольку требует оценки пороговой энергии $E_{\mathrm{th}}$ и интеграла (2) для каждого набора значений длины волны и глубины атмосферы, тогда как использование постоянного, не зависящего от $\lambda$ значения показателя преломления позволяет вычислять этот интеграл один раз для каждого значения глубины атмосферы.

В настоящей работе из процессов, отвечающих за убыль черенковских фотонов при распространении через атмосферу, были учтены следующие эффекты: а) поглощение черенковских фотонов атмосферным озоном (описывается коэффициентом $\mu_{\mathrm{O}_{3}}\left[\mathrm{~cm}^{-1}\right]$ ) и b) рэлеевское рассеяние (описывается коэффициентом $\mu_{R}\left[\mathrm{~cm}^{-1}\right]$ ). Общий коэффициент поглощения $\mu$, входящий в уравнение (1), определяется их суммой: $\mu=\mu_{\mathrm{O}_{3}}+\mu_{R}$.

Поглощение на атмосферном озоне является основным процессом, отвечающим за убыль черенковских фотонов ШАЛ в УФ диапазоне. Коэффициент $\mu_{\mathrm{O}_{3}}$ рассчитывался по формуле:

$$
\mu_{\mathrm{O}_{3}}=\sigma_{\mathrm{O}_{3}} N_{\mathrm{O}_{3}}=\sigma_{\mathrm{O}_{3}} \frac{P_{\mathrm{O}_{3}}}{k_{\mathrm{B}} T},
$$

где $\sigma_{\mathrm{O}_{3}}-$ сечение поглощения излучения озоном, $N_{\mathrm{O}_{3}}$ - концентрация атмосферного озона на высоте $h$, $P_{\mathrm{O}_{3}}$ - давление озона на высоте $h, k_{\mathrm{B}}$ - постоянная Больцмана, $T$ - температура на высоте $h$. Данные по сечению $\sigma_{\mathrm{O}_{3}}$ взяты из работ $[30,31]$. Количество атмосферного озона значительно изменяется в зависимости от местности, времени года и конкретных метеоусловий. Для реализации конкретных расчетов в настоящей работе были использованы экспериментальные данные по давлению $P_{\mathrm{O}_{3}}$ и температуре $T$ из исследования [32].

Следующим по значимости процессом, отвечающим за изменение потока черенковских фотонов ШАЛ, является рэлеевское рассеяние. Особенность описания переноса излучения с помощью уравнения (1) состоит в том, что рассматривается пучок с раствором $\simeq 5^{\circ}$. В то же время рэлеевское рассеяние обладает широким угловым распределением. Таким образом, можно считать, что каждый акт рэлеевского рассеяния приводит к выбыванию черенковского фотона из рассматриваемого пучка, что в уравнении (1) эффективно описывается как поглощение. Зависимость коэффициента рэлеевского рассеяния $\mu_{R}$ от глубины атмосферы (высоты) обусловлена изменением плотности атмосферы и дается следующим выражением:

$$
\mu_{R}(x, \lambda)=\mu_{R 0}(\lambda) \frac{\rho(x)}{\rho_{0}},
$$

где $\mu_{R 0}-$ коэффициент рэлеевского рассеяния на уровне моря. Для описания коэффициента рэлеевского 
рассеяния $\mu_{R 0}$ была использована аппроксимационная формула из работы Бучольца [28].

\section{3. Результаты моделирования и обсуждение}

Для моделирования наблюдаемых спектров черенковского излучения ШАЛ, уравнение (1) было численно проинтегрировано для различных значений энергии и типа первичной частицы, а также для различных высот наблюдения.

Результаты моделирования спектров излучения ШАЛ от энергичных $\gamma$ - и $p$-событий для высоты наблюдения $5 \mathrm{~km}$ представлены на рис. 1. Для более наглядного представления спектральных особенностей при $\lambda \lesssim 300 \mathrm{~nm}$, значения по горизонтальной оси ограничены величиной $400 \mathrm{~nm}$ (так как спектры в диапазоне 400-700 nm значимых особенностей не имеют и ведут себя монотонно согласно зависимости $\sim \lambda^{-2}$ ). Энергии первичных $\gamma$-квантов указаны рядом с кривыми. Следует отметить,

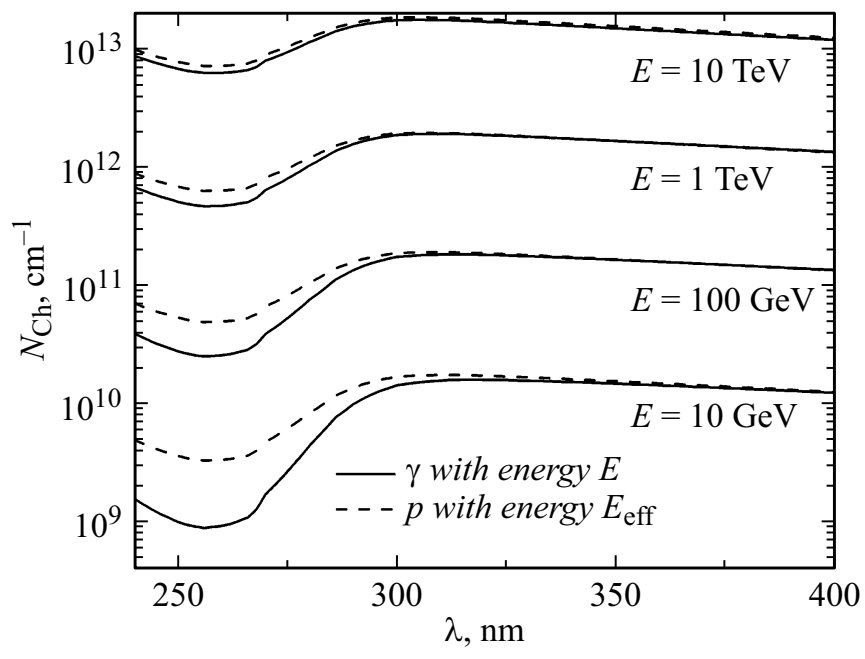

Рис. 1. Зависимость спектральной плотности $N_{\mathrm{Ch}}$ черенковского излучения ШАЛ от длины волны $\lambda$ для различных типов первичных частиц на высоте наблюдения $5 \mathrm{~km}$ над уровнем моря. Сплошные кривые соответствуют спектральной плотности черенковского излучения ШАЛ от $\gamma$-квантов с энергией $E$. Штриховые кривые соответствуют спектральной плотности черенковского излучения ШАЛ от протонов космических лучей с энергиями $E_{\mathrm{eff}}$ р расчитанными по формуле (23).

Параметры аппроксимации числа оптических фотонов по формуле (25)

\begin{tabular}{c|c|c}
\hline $\begin{array}{c}\text { Тип частицы, } \\
\text { высота наблюдения }\end{array}$ & $N_{10}, 10^{5}$ & $\xi$ \\
\hline$\gamma, 2 \mathrm{~km}$ & 3.174 & 0.044 \\
$p, 2 \mathrm{~km}$ & 1.894 & 0.07 \\
$\gamma, 5 \mathrm{~km}$ & 3.388 & 0.03 \\
$p, 5 \mathrm{~km}$ & 1.868 & 0.04
\end{tabular}

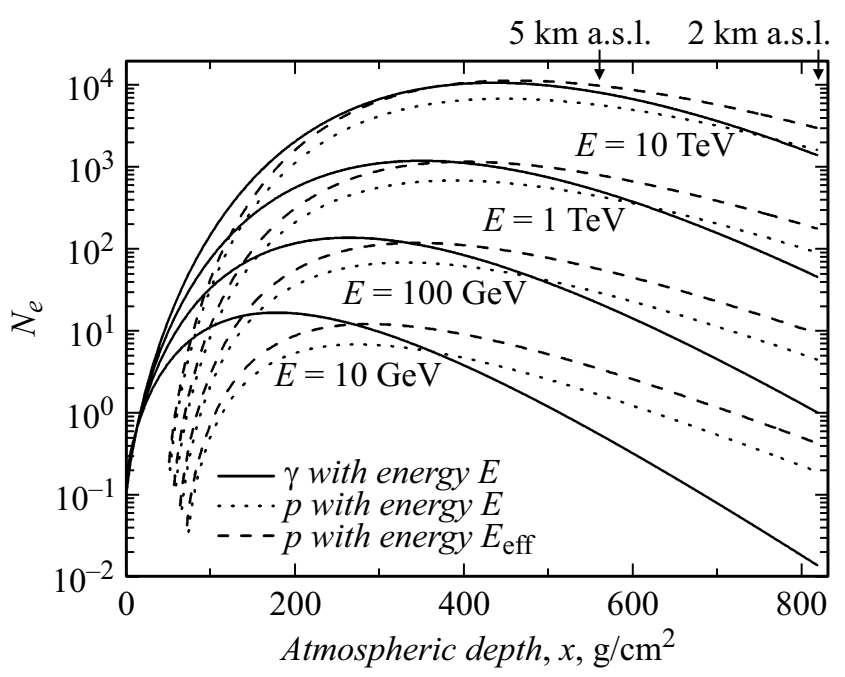

Рис. 2. Зависимость среднего количества электронов ШАЛ $N_{e}$ от глубины атмосферы $x$ (продольные [высотные] профили ШАЛ) для различных типов первичных частиц. Сплошные кривые соответствуют ШАЛ от $\gamma$-квантов с энергией $E$ (указана рядом с соответствующими кривыми), точечные кривые соответствуют ШАЛ от протонов космических лучей с энергиями $E$, штриховые кривые соответствуют ШАЛ от протонов космических лучей с энергиями $E_{\text {eff, }}$ рассчитанными по формуле (23).

что спектры черенковского излучения рШАЛ при этом получены для энергий первичных протонов

$$
E_{\text {eff }} \simeq 17.73\left(\frac{E}{10 \mathrm{GeV}}\right)^{0.99}
$$

которые определяются из условия равного количества черенковских фотонов

$$
N_{\mathrm{opt}}=\int N_{\mathrm{Ch}} d \lambda
$$

в диапазоне 320-700 nm, условно именуемом ниже „оптическим“. 2 Количество оптических фотонов, излученных ШАЛ, может быть примерно ${ }^{3}$ определено по следующей формуле:

$$
N_{\mathrm{opt}}=N_{10}\left(\frac{E}{10 \mathrm{GeV}}\right)^{1+\xi}
$$

где параметры $N_{10}$ (количество оптических фотонов при энергии первичной частицы $10 \mathrm{GeV}$ ) и $\xi$ приведены в таблице.

Сравнение аппроксимационных параметров из таблицы показывает, что при равных энергиях первичных

\footnotetext{
2 Поскольку бо́льшая часть указанного диапазона соответствует стандартному диапазону V (но также охватывает диапазон UVA) и в нем обладают заметной чувствительностью наиболее широко используемые в настоящее время SiPM (например, Hamamatsu S10362$33[29])$.

${ }^{3} \mathrm{C}$ точностью около 6\% для первичных частиц с энергией менее $1 \mathrm{TeV}$ на высоте наблюдения $5 \mathrm{~km}$ и с точностью лучше $4 \%$ для первичных частиц с энергией менее $3 \mathrm{TeV}$ на высоте наблюдения $2 \mathrm{~km}$.
} 
частиц в рШАЛ образуется меньше черенковских фотонов, чем в $\gamma$ ШАЛ, а генерация равного количества оптических фотонов ШАЛ требует бо́льшей энергии протонов. В основном это объясняется тем, что в рШАЛ генерируется меньше электронов, чем в $\gamma$ ШАЛ, что, в свою очередь, ведет к образованию меньшего количества черенковских фотонов. Различие в количестве электронов ШАЛ можно наглядно увидеть из отношения формул (12) и (17):

$$
\frac{N_{e, \max }^{\gamma}(E)}{N_{e, \max }^{p}(E)} \simeq \frac{5.34}{\sqrt{\ln \left(E / E_{\gamma c}\right)}} .
$$

Это отношение меняется от $\simeq 2.4$ до $\simeq 1.6$ в диапазоне энергий $10 \mathrm{GeV}-10 \mathrm{TeV}$ (см. также рис. 2). Общее количество сгенерированных ШАЛ электронов также зависит от протяженности (ширины профиля, рис. 2) и глубины залегания области генерации в атмосфере, поскольку преломляющая способность $\Delta n$ экспоненциально зависит от высоты.

Наличие максимума спектральной плотности $N_{\mathrm{Ch}}$ черенковского излучения ШАЛ при $\lambda=300-330 \mathrm{~nm}$ и ее резкое уменьшение при $\lambda \lesssim 300 \mathrm{~nm}$ обусловлено существенным поглощением излучения атмосферным озоном. Рис. 1 иллюстрирует следующие закономерности: 1) при равном количестве оптических фотонов черенковского излучения ШАЛ количество фотонов УФ диапазона $(\lambda \leq 300 \mathrm{~nm})$ может быть значительно больше в случае, если первичной частицей был протон; 2) с увеличением энергии первичной частицы доля УФ фотонов в спектре излучения $\gamma$ ШАЛ существенно растет (что видно из сравнения величины минимума $\lambda \simeq 260 \mathrm{~nm}$ и максимума $\lambda \simeq 310 \mathrm{~nm}$ спектральной плотности для каждой кривой), а отличие спектров $\gamma$ ШАЛ и рШАЛ уменьшается.

Эти закономерности могут быть поняты при детальном рассмотрении зависимостей (9) и (13) - так называемых „продольных профилей ШАЛ“, - описывающих развитие электрон-фотонных каскадов ШАЛ (рис. 2). Видно, что для первичных частиц с энергиями $\lesssim 100 \mathrm{GeV}$ максимум количества электронов ШАЛ, область значительного количества электронов ШАЛ [например, $N_{e} \gtrsim 0.1 N_{e, \max }$ ] в целом и, соответственно, область эффективной генерации черенковского излучения ШАЛ, в среднем находятся глубже, если первичной частицей является протон с энергией $E_{\text {eff, }}$ чем в случае, если первичной частицей является $\gamma$-квант с энергией $E$. Это приводит к тому, что оптическая толща по взаимодействию с атмосферным озоном, которую проходит черенковское излучение в случае рШАЛ, оказывается меньше, чем в случае $\gamma$ ШАЛ. В диапазоне $\lambda \gtrsim 320 \mathrm{~nm}$, где поглощение на озоне мало (т.е. атмосфера практически прозрачна для излучения) и оптическая толща много меньше единицы $\left(\lesssim 10^{-2}\right)$ это не приводит к каким-либо значительным отличиям в спектре черенковского излучения ШАЛ. Но в диапазоне $\lambda \lesssim 300 \mathrm{~nm}$, где поглощение становится существенным (вследствие практически

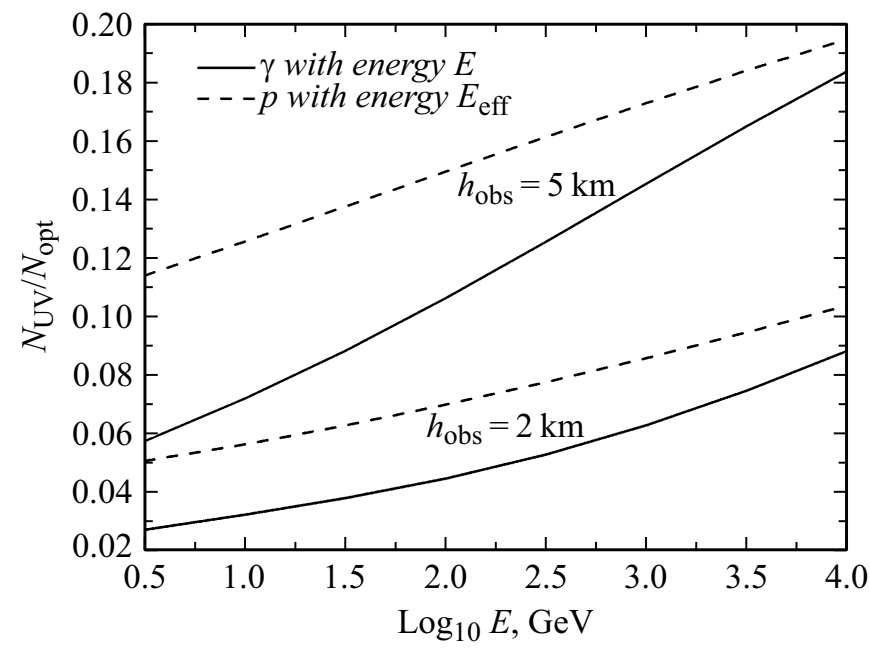

Рис. 3. Отношение $N_{\mathrm{UV}} / N_{\mathrm{opt}}$ количества фотонов черенковского излучения ШАЛ в УФ диапазоне $(240-300 \mathrm{~nm})$ к количеству фотонов черенковского излучения ШАЛ в оптическом диапазоне $(320-700 \mathrm{~nm})$ в зависимости от энергии первичной частицы для различных типов первичных частиц и высот наблюдения (указаны рядом с соответствующими парами кривых). Сплошные кривые соответствуют $N_{\mathrm{UV}} / N_{\mathrm{opt}}$ для ШАЛ от $\gamma$-квантов с энергией $E$, штриховые - ШАЛ от протонов космических лучей с энергиями $E_{\text {eff }}$, рассчитанными по формуле (23).

экспоненциального роста сечения с уменьшением длины волны (см., например [30,31])) и особенно в диапазоне $\lambda \lesssim 280 \mathrm{~nm}$, где оптическая толща по поглощению на озоне становится велика ( $\gtrsim 1)$, разница между величинами оптического пути черенковского излучения $\gamma$ ШАЛ и рШАЛ приводит к значительным (в разы) отличиям в величине спектральной плотности $N_{\mathrm{Ch}}$.

С ростом энергии первичных частиц отличия в продольных профилях $\gamma$ ШАЛ и рШАЛ уменьшаются (сравни кривые для $E \geq 1 \mathrm{TeV}$ на рис. 2), что приводит к формированию значительно более близких спектров черенковского излучения ШАЛ для первичных фотонов и протонов с одинаковой эффективной энергией. Также из рис. 2 и формул (10), (14) видно, что глубина залегания области эффективной генерации черенковского излучения ШАЛ растет с ростом энергии первичной частицы, что, очевидно, приводит к уменьшению оптической толщи по поглощению на озоне, и, соответственно, к росту доли УФ излучения в спектре черенковского излучения ШАЛ.

Для количественной оценки доли УФ излучения в спектре черенковского излучения ШАЛ можно использовать величину $N_{\mathrm{UV}} / N_{\mathrm{opt}}$, где $N_{\mathrm{UV}}=\int N_{\mathrm{Ch}} d \lambda-$ количество фотонов в диапазоне $\lambda=240-300 \mathrm{~nm}$, который ниже условно именуется „ультрафиолетовым“. 4 Зависимости отношения $N_{\mathrm{UV}} / N_{\text {opt }}$ от эквивалентной энергии $E$ первичной частицы (т. е. для энергии $\gamma$-кванта $E$ и энергии протона $\left.E_{\text {eff }}\right)$ для различных типов первичных частиц

\footnotetext{
${ }^{4}$ Он охватывает бо́льшую часть диапазона MUV.
} 
на разных высотах наблюдения приведены на рис. 3. Вид этих кривых подтверждает и конкретизирует качественные выводы, сделанные выше на основании рис. 1. Для обеих высот наблюдения отношение $N_{\mathrm{UV}} / N_{\text {opt }}$ pacтет с ростом энергии первичной частицы, и значение отношения $N_{\mathrm{UV}} / N_{\text {opt }}$ для $\gamma$-квантов с ростом энергии приближается к значению $N_{\mathrm{UV}} / N_{\text {opt }}$ для протонов. Рис. 3 позволяет дополнительно сделать следующие важные выводы: 1) на высоте наблюдения $5 \mathrm{~km}$ доля УФ фотонов в спектре черенковского излучения ШАЛ примерно в два раза выше, чем на высоте $2 \mathrm{~km} ; 2)$ на высоте наблюдения $5 \mathrm{~km}$ значение величины $\left(N_{\mathrm{UV}} / N_{\text {opt }}\right)_{p} /\left(N_{\mathrm{UV}} / N_{\text {opt }}\right)_{\gamma}$ составляет 2-1.4 при эквивалентной энергии первичной частицы менее $100 \mathrm{GeV}$ и падает до значения $\simeq 1.05$ при эквивалентной энергии первичной частицы $10 \mathrm{TeV}$; 3) на высоте наблюдения $2 \mathrm{~km}$ значение величины $\left(N_{\mathrm{UV}} / N_{\mathrm{opt}}\right)_{p} /\left(N_{\mathrm{UV}} / N_{\mathrm{opt}}\right)_{\gamma}$ составляет примерно $1.9-1.6$ при эквивалентной энергии первичной частицы менее $100 \mathrm{GeV}$ и падает до значения $\simeq 1.2$ при эквивалентной энергии первичной частицы $10 \mathrm{TeV}$. Таким образом, если в качестве критерия идентификации типа первичной частицы принимать значение наблюдаемого отношения $N_{\mathrm{UV}} / N_{\text {opt}}$, то можно сказать, что этот метод может быть с равным успехом применим как на высоте наблюдения $2 \mathrm{~km}$, так и на высоте $5 \mathrm{~km}$ при эквивалентных энергиях первичных частиц менее $100 \mathrm{GeV}$. На высоте $5 \mathrm{~km}$ при эквивалентной энергии первичных частиц выше $1 \mathrm{TeV}$ метод становится практически бесполезен, в то время как на высоте $2 \mathrm{~km}$ его эффективность при энергиях выше $1 \mathrm{TeV}$, хотя и падает, но тем не менее, он еще может быть использован в качестве дополнительного способа повышения надежности идентификации типа первичной частицы. При этом, однако, следует учитывать следующие обстоятельства:

1) в диапазоне энергий выше $100 \mathrm{GeV}$, где количество регистрируемых черенковских фотонов велико $\left(\gtrsim 10^{3}\right)$, достаточно надежным методом идентификации типа первичной частицы является морфологический анализ на основе параметров Хилласа, широко применяемый в современной черенковской гамма-астрономии. Идентификация первичной частицы является более сложной на низких энергиях $(\lesssim 30 \mathrm{GeV})$, где количество регистрируемых черенковских фотонов значительно меньше. $\mathrm{C}$ этой точки зрения, реализация метода идентификации первичной частицы по значению величины $N_{\mathrm{UV}} / N_{\text {opt }}$ может играть важную роль именно для наблюдений на низких энергиях;

2) для успешной реализации упомянутого метода анализа УФ компоненты черенковского излучения ШАЛ необходимо не только наличие достаточной чувствительности спектра к типу первичной частицы, но и достаточное для регистрации количество черенковских фотонов в диапазоне, где спектр чувствителен к типу первичной частицы. С этой точки зрения, реализация наблюдения УФ компоненты черенковского излучения ШАЛ на высоте $5 \mathrm{~km}$ выглядит значительно более перспективной, чем на высоте $2 \mathrm{~km}$, поскольку количество
УФ фотонов на высоте $5 \mathrm{~km}$ в два раза больше при прочих равных условиях.

\section{1. Детектирование УФ излучения ШАЛ на телескопе ГТ-48}

Важным примером практического применения фотодетекторов с чувствительностью в УФ диапазоне совместно с фотодетекторами оптического диапазона является схема, реализованная на черенковском телескопе второго поколения ГТ-48 Крымской обсерватории $\left(h_{\mathrm{obs}} \simeq 600 \mathrm{~m}\right.$ над уровнем моря), работавшем в тераэлектроновольтном диапазоне энергий первичных частиц $[4,3]$. Использованные на ГТ-48 фотодетекторы УФ диапазона обладали чувствительностью в диапазоне 200-300 nm, фотодетекторы оптического диапазона обладали чувствительностью в диапазоне 330-600 nm. Были проведены измерения отношения количества УФ фотонов к количеству оптических фотонов черенковского излучения ШАЛ $\left(N_{\mathrm{UV}} / N_{\mathrm{opt}}\right)^{\mathrm{GT} 48}$ (индекс GT48 показывает, что величина относится к работе [4]) и разработаны соответствующие критерии сепарации типов первичных частиц. Такой метод был успешно применен для повышения достоверности определения типа первичной частицы при наблюдении космических источников гамма-излучения (например, $[33,34])$. Типичные значения измеренного [4] отношения $\left(N_{\mathrm{UV}} / N_{\mathrm{opt}}\right)^{\mathrm{GT} 48}$ составили 0.04-0.06 при энергии первичных частиц $4 \mathrm{TeV}$. С учетом отличия в определениях УФ и оптического диапазонов, использованных [4], где УФ диапазон шире, а оптический диапазон у́же, чем в настоящей работе, а также с учетом отличия в высоте наблюдения, можно утверждать, что результаты настоящей работы (рис. 3) хорошо согласуются с результатами [4].

\section{2. Моделирование УФ излучения ШАЛ группой Рахмана}

Помимо практической реализации метода гаммаадронной сепарации на основе измерения отношения количества УФ фотонов к количеству оптических фотонов черенковского излучения ШАЛ на телескопе ГТ48 , существуют теоретические исследования вопроса о чувствительности спектра черенковского излучения ШАЛ к типу первичной частицы. Величина, аналогичная рассмотренному отношению $N_{\mathrm{UV}} / N_{\text {opt }}$, была рассчитана в работе Рахмана и др. [8] для несколько иных диапазонов длин волн: под оптическим диапазоном понимался интервал 300-550 nm, под УФ диапазоном - интервал 270-300 nm. Расчеты выполнялись для высот наблюдения до $2 \mathrm{~km}$ и диапазона энергий первичных частиц $50 \mathrm{GeV}-3 \mathrm{TeV}$. Эти отличия затрудняют детальное сравнение результатов [8] с результатами настоящей работы. Так, отношение $\left(N_{\mathrm{UV}} / N_{\text {opt }}\right)^{\mathrm{Rahm}}$ (индекс „Rahm“ обозначает, что значение относится к работе [8]), рассчитанное для высоты $2 \mathrm{~km}$, составляет 8.29-8.44\% для ШАЛ от $\gamma$-квантов с энергиями $50-1000 \mathrm{GeV}$ и $9.77-9.91 \%$ для 


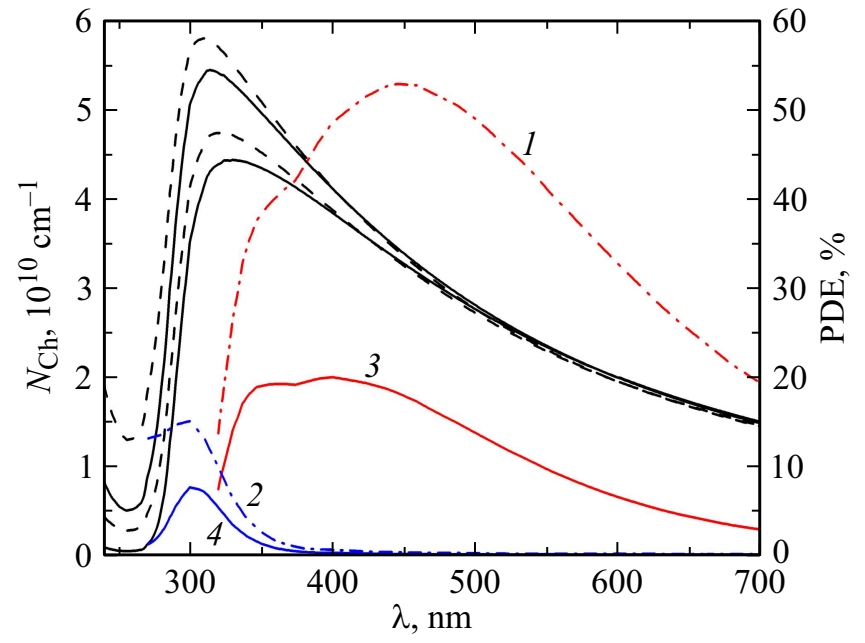

Рис. 4. Зависимости спектральной плотности $N_{\mathrm{Ch}}$ черенковского излучения ШАЛ (значения соответствуют левой оси графика) и эффективности детектирования PDE (значения соответствуют правой оси графика) от длины волны $\lambda$. Черные сплошные кривые соответствуют ШАЛ от $\gamma$-квантов с энергией $\simeq 31.6 \mathrm{GeV}$, черные штриховые кривые соответствуют ШАЛ от протонов космических лучей с энергиями $\sim 49.8 \mathrm{GeV}$ (для высоты наблюдения $2 \mathrm{~km}$ ) и $\simeq 55.4 \mathrm{GeV}$ (для высоты наблюдения $5 \mathrm{~km}$ ). Верхняя пара кривых соответствует высоте наблюдения $5 \mathrm{~km}$, нижняя - $2 \mathrm{~km}$. Кривая (1) описывает эффективность фотодетекторов Hamamatsu Photonics s1036233, кривая (2) описывает эффективность фотодетекторов, разработанных в ФТИ им. А.Ф. Иоффе [17]. Дополнительно показаны кривые (значения соответствуют левой оси графика), описывающие количество фотонов $N_{\mathrm{Ch}}(\lambda) \cdot \operatorname{PDE}_{X}(\lambda)$, которые могут быть зарегистрированы с помощью фотодетекторов Hamamatsu Photonics s10362-33 (3) и с помощью фотодетекторов, разработанных в ФТИ им. А.Ф. Иоффе [17], (4) на высоте $5 \mathrm{~km}$ от ШАЛ, вызванного $\gamma$-квантом с энергией $\simeq 31.6 \mathrm{GeV}$.

ШАЛ от протонов с энергиями 100-2000 GeV. Разница величин $N_{\mathrm{UV}} / N_{\text {opt }}$ и $\left(N_{\mathrm{UV}} / N_{\mathrm{opt}}\right)^{\text {Rahm }}$ объясняется именно отличием в определении используемых диапазонов: ${ }^{5}$ так как используемый в настоящей работе оптический диапазон значительно шире, то в нем при прочих равных условиях находится на $15-20 \%$ больше черенковских фотонов, чем в диапазоне, использованном [8]. Таким образом результаты расчетов величины $N_{\mathrm{UV}} / N_{\text {орt}}$, представленные в настоящей работе, не противоречат результатам [8]. Также, с учетом разницы в спектральных диапазонах и высотах наблюдения (в работе [8] эти данные приведены для высоты наблюдения $1 \mathrm{~km}$ ), удовлетворительно согласуются интегральные количества фотонов оптического диапазона.

Некоторые отличия расчетных отношений $N_{\mathrm{UV}} / N_{\mathrm{opt}}$ также объясняются следующими различиями в матема-

\footnotetext{
${ }^{5}$ Следует отметить, что учет в настоящей работе черенковского излучения ШАЛ с длинами волн вплоть до $700 \mathrm{~nm}$ имеет важное значение, поскольку современные фотодетекторы оптического диапазона, планирующиеся к использованию на черенковских гамма-телескопах нового поколения, обладают значительной чувствительностью на таких длинах волн.
}

тических моделях, использованных в настоящей работе и в работе [8]: 1) для распределения электронов ШАЛ по энергии Рахман и др. [8] использовали формулу из работы Зацепина и Чудакова $[35],{ }^{6}$ в то время как в настоящей работе использована более точная формула Гиллера и др. [22], которая представляется более подходящей для описания распределения электронов ШАЛ; 2) для описания продольных профилей ШАЛ от протонов Рахман и др. [8] использовали приближение Гайссера-Хилласа [36], в то время как в настоящей работе для этой цели использовано приближение Грейзена-Ильиной-Линсли $[23,19] ; 3)$ для описания изменения преломляющей способности с высотой (глубиной атмосферы) в работе [8] использована простая экспоненциальная зависимость от высоты (что не просто неточно, но и плохо согласуется с использованной в той же работе зависимостью высоты от глубины атмосферы), в то время как в настоящей работе использована более обоснованная зависимость на основе соотношения Гладстона-Дэйла (20) и зависимости плотности от глубины атмосферы (19). Кроме того, качественное отличие и новизна настоящего исследования по сравнению с работой [8] заключаются в том, что здесь впервые были проведены расчеты черенковских спектров ШАЛ и величины $N_{\mathrm{UV}} / N_{\text {opt }}$ для высоты $5 \mathrm{~km}$, актуальные в свете возможности размещения черенковских гаммаобсерваторий нового поколения именно на таких высотах над уровнем моря.

\section{3. Регистрация УФ излучения ШАЛ с помощью лавинных фотоумножителей SiPM: перспективы для проекта ALEGRO}

Значительный интерес представляет вопрос о том, насколько возможна регистрация УФ компоненты черенковского излучения ШАЛ и насколько целесообразно ее использование для определения типа первичной частицы с помощью серийных фотодетекторов с известной кривой спектральной чувствительности. Для ответа на этот вопрос нами были рассчитаны количества черенковских фотонов, которые могут быть зарегистрированы с помощью SiPM s10362-33 производства Hamamatsu Photonics и с помощью УФ чувствительных SiPM, разработанных в ФТИ им. А.Ф. Иоффе [17]. Кривые чувствительности этих SiPM (photon detection efficiency, PDE) представлены на рис. 4 вместе со спектрами черенковского излучения ШАЛ от первичного $\gamma$-кванта с энергией $\simeq 31.6 \mathrm{GeV}$ и первичных протонов с энергией $\simeq 49.8 \mathrm{GeV}$ (для высоты наблюдения $2 \mathrm{~km}$ ) и $\simeq 55.4 \mathrm{GeV}$ (для высоты наблюдения $5 \mathrm{~km}$ ). Из рис. 4 видно, что $\mathrm{SiPM}$ s10362-33 обладают чувствительностью в диапазоне $\lambda \gtrsim 320 \mathrm{~nm}$, т. е. в стандартном оптическом и UVA диапазонах, в то время как SiPM производства ФТИ обладают чувствительностью в диапазоне $\lambda \lesssim 350 \mathrm{~nm}$.

\footnotetext{
6 При этом, по всей видимости, формула была позаимствована неточно: использована ошибочная степень в зависимости плотности вероятности от энергии.
} 


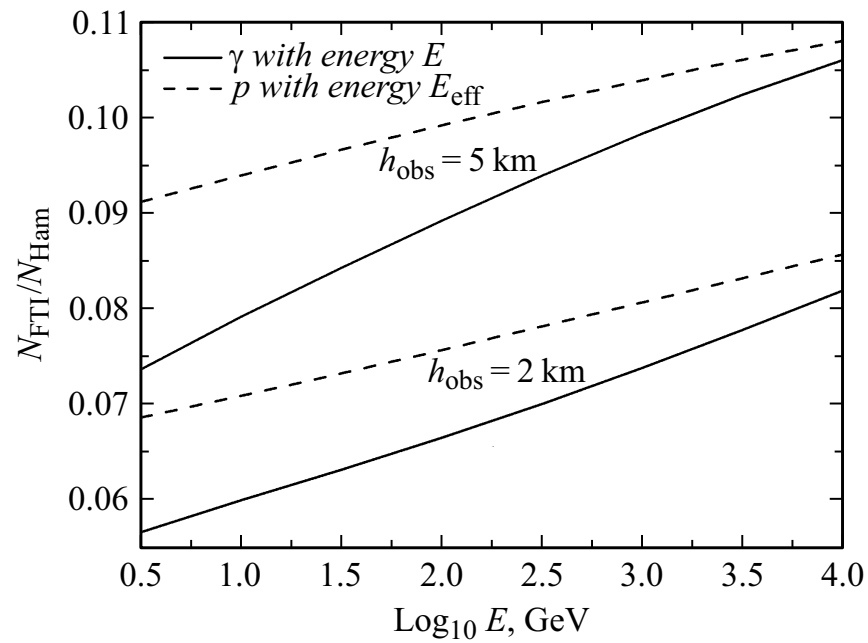

Рис. 5. Отношение $N_{\text {FтI }} / N_{\text {Hат }}$ количества фотонов черенковского излучения ШАЛ, которые могут быть зарегистрированы фотодетекторами, разработанными в ФТИ им. А.Ф. Иоффе [17], к количеству фотонов черенковского излучения ШАЛ, которые могут быть зарегистрированы фотодетекторами Hamamatsu Photonics s10362-33, в зависимости от энергии первичной частицы для различных типов первичных частиц и высот наблюдения (указаны рядом с соответствующими парами кривых). Сплошные кривые соответствуют $N_{\text {FтI }} / N_{\text {Hат }}$ для ШАЛ от $\gamma$-квантов с энергией $E$, штриховые - ШАЛ от протонов космических лучей с энергиями $E_{\text {eff, }}$ рассчитанными по формуле (23).

Таким образом, при одной и той же величине сигнала в камере, построеной на SiPM Hamamatsu, величина сигнала в камере, построеной на SiPM производства ФТИ, будет отличаться в зависимости от типа первичной частицы и высоты наблюдения. Количество черенковских фотонов ШАЛ, которые могут быть зарегистрированы при использовании соответствующих SiPM, в общем количестве черенковских фотонов ШАЛ может быть определено как

$$
N_{X}=\int_{0}^{\infty} N_{\mathrm{Ch}}(\lambda) \operatorname{PDE}_{X}(\lambda) d \lambda,
$$

где индекс $X$ принимает значения „FTI“ для SiPM производства ФТИ и „Нам“ для SiPM производства Hamamatsu.

Результаты расчетов отношения $N_{\text {FтI }} / N_{\text {Ham }}$ в зависимости от эквивалентной энергии $E$ первичной частицы (то есть, для энергии $\gamma$-кванта $E$ и энергии протона $\left.E_{\text {eff }}\right)$, для различных типов первичных частиц приведены на рис. 5. Отношение $N_{\text {FтI }} / N_{\text {Hаm }}$ демонстрирует те же тенденции, что и отношение $N_{\mathrm{UV}} / N_{\text {opt }}$ : для обеих высот наблюдения отношение $N_{\mathrm{FTI}} / N_{\text {Ham }}$ растет с ростом энергии первичной частицы (вне зависимости от ее типа), и значение отношения $N_{\text {FтI }} / N_{\text {Ham }}$ для $\gamma$ квантов с ростом энергии приближается к значению $N_{\mathrm{FTI}} / N_{\mathrm{Ham}}$ для протонов. Рис. 5 также позволяет сделать следующие выводы: 1) на высоте наблюдения $5 \mathrm{~km}$ отношение $N_{\text {FTI }} / N_{\text {Ham }}$ примерно на $30 \%$ больше, чем на высоте $2 \mathrm{~km}$, при сигналах одинаковой мощности от фотодетекторов Hamamatsu s10362-33, фотодетекторы ФТИ будут регистрировать на $30 \%$ больше черенковских фотонов от $\gamma$ ШАЛ и на $33-26 \%$ больше черенковских фотонов от рШАЛ на высоте $5 \mathrm{~km}$, чем на высоте $2 \mathrm{~km}$; 2) на высоте наблюдения $5 \mathrm{~km}$ значение величины $\left(N_{\mathrm{FTI}} / N_{\mathrm{Ham}}\right)_{p} /\left(N_{\mathrm{FTI}} / N_{\mathrm{Ham}}\right)_{\gamma}$ составляет примерно 1.2-1.1 при эквивалентной энергии первичной частицы менее $100 \mathrm{GeV}$ и падает до значения $\simeq 1.02$ при эквивалентной энергии первичной частицы $10 \mathrm{TeV}$; 3) на высоте наблюдения $2 \mathrm{~km}$ значение величины $\left(N_{\mathrm{FTI}} / N_{\mathrm{Ham}}\right)_{p} /\left(N_{\mathrm{FTI}} / N_{\mathrm{Ham}}\right)_{\gamma}$ также составляет примерно $1.2-1.1$ при эквивалентной энергии первичной частицы менее $100 \mathrm{GeV}$ и падает до значения $\simeq 1.05$ при эквивалентной энергии первичной частицы $10 \mathrm{TeV}$.

C учетом невысокой чувствительности величины $N_{\text {FTI }} / N_{\text {Hаm }}$ к типу первичной частицы $(10-20 \%)$, сложно ожидать, что после полноценного численного многочастичного моделирования методом Монте-Карло (позволяющего, в частности, оценить флуктуации величин, характеризующих ШАЛ), можно будет выработать надежные критерии гамма-адронной сепарации с помощью измерения УФ компоненты и использовать такой анализ как единственный метод определения типа первичной частицы с применением уже существующих детекторов. Вместе с тем, полученные результаты демонстрируют возможность использования анализа УФ компоненты 240-300 nm совместно с иными методами (в первую очередь, с анализом параметров Хилласа) как дополнительного подхода, повышающего достоверность определения типа частицы. В то же время чувствительность величины $N_{\mathrm{UV}} / N_{\text {opt }}$ к типу первичной частицы $(40-100 \%)$ позволяет надеятся, что в случае оптимизации SiPM под требования данной задачи (в частности, смещения основной чувствительности в диапазон $\lesssim 300-320 \mathrm{~nm}$ и увеличения эффективности детектирования), метод определения типа первичной частицы посредством измерения количества УФ фотонов по отношению к количеству оптических фотонов черенковского излучения ШАЛ может стать весьма эффективным.

Поскольку при стандартной схеме детекторной камеры черенковского телескопа использование УФ детекторов так или иначе происходит за счет замещения ими детекторов оптического диапазона, а полный полезный сигнал в УФ диапазоне заведомо ниже, чем в оптическом диапазоне, это может привести к ухудшению некторых показателей черенковской обсерватории, в частности, к увеличению пороговой энергии наблюдений. И хотя это будет до некоторой степени компенсироваться улучшением других показателей (например, эффективности гамма-адронной сепарации ШАЛ), тем не менее, возникает вопрос о том, какая еще полезная информация может быть извлечена из наблюдений УФ компоненты черенковского излучения ШАЛ, и какие еще параметры обсерватории могут быть улучшены за счет этого. 
Одной из очевидных возможностей получения полезной информации из УФ сигнала является возможность построения изображений черенковских вспышек ШАЛ, аналогично тому, как это делается при обработке сигнала в оптическом диапазоне, и последующее применение стандартного анализа морфологических признаков полученных изображений путем оценки параметров Хилласа. Такой анализ может быть весьма успешно проведен, если количество зарегистрированных фотонов $N_{\text {reg }} \gtrsim N_{\text {crit }} \simeq 1.3 \cdot 10^{2}$ (это условие выполняется, например, при наблюдении телескопом с зеркалом диаметром $30 \mathrm{~m}$ оптического черенковского излучения ШАЛ от $\gamma$-квантов с энергией $5 \mathrm{GeV}$ на высоте $5 \mathrm{~km}[15])$. При известных параметрах черенковского гамма-телескопа и его детекторных камер, это требование позволяет оценить пороговую энергию регистрации $E_{0}$, используя следующее соотношение:

$$
\begin{aligned}
N_{\text {crit }} & \simeq S_{\text {tel }} \int_{0}^{\infty} \frac{N_{\mathrm{Ch}}\left(E_{0}, \lambda\right)}{S_{\mathrm{EAS}}} R(\lambda) \operatorname{PDE}_{X}(\lambda) d \lambda \\
& \simeq \frac{S_{\text {tel }}}{S_{\mathrm{EAS}}} \bar{R} N_{X}\left(E_{0}\right),
\end{aligned}
$$

где $S_{\text {tel }}$ - площадь собирающей поверхности зеркала телескопа, параметр $S_{\mathrm{EAS}}$ по порядку величины совпадает с типичной площадью поперечного сечения $\gamma$ ШАЛ, $R(\lambda)$ - отражающая способность зеркала, $\bar{R}-$ средняя по спектру отражающая способность зеркала. Здесь предполагается, что отражающая способность зеркала телескопа в УФ диапазоне может быть достаточно большой $(R(\lambda) \gtrsim 80 \%$ - см., например, [37]). Для оценки использовано значение средней отражающей способности зеркала $\bar{R}=0.8$. Зависимость спектральной плотно-

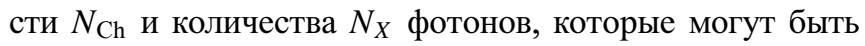
зарегистрированы с использованием фотодетекторов типа $X$, от энергии первичной частицы указана явно, чтобы показать, каким именно образом соотношение (28) позволяет определить пороговую энергию наблюдений. Значение параметра $S_{\text {EAS }}$ подбирается из условия согласования значения поверхностной плотности черенковских фотонов $\gamma$ ШАЛ $\rho_{\text {opt }}=N_{\text {opt }} / S_{\text {EAS на расстоянии }}$ порядка $100 \mathrm{~m}$ от оси (ствола) ШАЛ от $\gamma$-квантов с энергией $30 \mathrm{GeV}$ с результатами многочастичных расчетов, выполненных методом Монте-Карло (например, $[14,15])$. Исходя из этого условия, для высоты $5 \mathrm{~km}$ $S_{\mathrm{EAS}} \simeq 2 \cdot 10^{5} \mathrm{~m}^{2}$, а для высоты $2 \mathrm{~km} S_{\mathrm{EAS}} \simeq 3.3 \cdot 10^{5} \mathrm{~m}^{2}$. Проект черенковской гамма-обсерватории ALEGRO [16] предполагает, что в ее телескопах будут использованы зеркала диаметром $30 \mathrm{~m}$, т.е. площадь зеркала будет составлять $S_{\text {tel }} \simeq 700 \mathrm{~m}^{2}$. Таким образом, при использовании фотодетекторов производства ФТИ [17] пороговая энергия регистрации гамма-событий в обсерватории ALEGRO определяется следующими условиями: на высоте $5 \mathrm{~km} N_{\mathrm{FTI}}\left(E_{0}\right) \simeq 4.6 \cdot 10^{4}$, что соответствует значению $E_{0} \simeq 37 \mathrm{GeV}$; на высоте $2 \mathrm{~km} N_{\mathrm{FTI}}\left(E_{0}\right) \simeq 7.6 \cdot 10^{4}$, что соответствует значению $E_{0} \simeq 80 \mathrm{GeV}$.

\section{4. Детектирование УФ излучения ШАЛ на телескопе Whipple}

Еще одним важным практическим примером наблюдений на черенковском гамма-телескопе, осуществленных с помощью камер, чувствительных в УФ диапазоне, являются наблюдения Крабовидной туманности и блазара Mrk421, выполненные в обсерватории Fred Lawrence Whipple Observatory на телескопе Whipple (был установлен на Mt. Hopkins, $h_{\text {obs }}=2.3 \mathrm{~km}$ a.s.1., площадь зеркала - $75 \mathrm{~m}^{2}$ ). Важность этого примера в контексте настоящей работы заключается в том, что для наблюдений использовались только камеры, чувствительные в УФ диапазоне, с последующим анализом морфологических признаков полученных изображений (т.е. одновременные наблюдения черенковского излучения ШАЛ в оптическом диапазоне не производились). Такая конфигурация позволяет оценить величину пороговой энергии, характерную именно для наблюдений в УФ диапазоне.

Конкретно, наблюдения Крабовидной туманности с помощью УФ камеры на основе фотоумножителей Hamamatsu R1802 показали, что пороговая энергия наблюдений составила $1.2 \mathrm{TeV}[5,38]$. Наблюдения Крабовидной туманности, выполненные на том же телескопе с помощью гибридной камеры из оптических фотоумножителей Hamamatsu R1398 с высокой эффективностью детектирования в диапазоне длин волн выше $250 \mathrm{~nm}$ и блокирующим фильтром 300-500 nm [6], показали, что пороговая энергия наблюдений составила $0.7 \mathrm{TeV}$. Разница в величине пороговой энергии в этих двух случаях была обусловлена тем, что оптические фотоумножители Hamamatsu R1398 обладали более высокой эффективностью детектирования в диапазоне 250-300 nm, чем УФ фотоумножители Hamamatsu R1802, несмотря на то, что такой диапазон не является их основным рабочим диапазоном. Можно сделать вывод, что рассчитанная нами величина пороговой энергии гамма-телескопа ALEGRO при использовании УФ фотодетекторов ФТИ $(80 \mathrm{GeV}$ на высоте $2 \mathrm{~km}$ ) согласуется со значениями, полученными в работах [5,6,38]. Разница в абсолютных значениях величины пороговой энергии полностью объясняется тем, что принятая в расчет площадь зеркал телескопов ALEGRO (около $700 \mathrm{~m}^{2}$ ) более чем в 9 раз выше площади зеркала телескопа Whipple, а также тем, что диапазоны преимущественной эффективности детектирования фотоумножителей Hamamatsu (с учетом использования блокирующего фильтра в гибридной камере) отличаются от рабочего диапазона фотодетекторов ФТИ.

\section{5. Возможности детектирования УФ излучения ШАЛ в проекте ALEGRO}

Планируемая величина пороговой энергии гаммаобсерватории ALEGRO при использовании оптических фотодетекторов (например, Hamamatsu s10362-33) coставляет около $5 \mathrm{GeV}$ [14-16]. Таким образом, при применении только УФ детекторов пороговая энергия 
вырастет (как это было экспериментально показано в работах $[5,6,10,11,38])$, но взамен обсерватория получит иные преимущества. Среди них можно выделить следующие.

1) Возможность проводить наблюдения в лунные ночи, что недоступно большинству современных черенковских гамма-телескопов. По оценкам [7] и [12] это может позволить увеличить длительность рабочего цикла гамма-обсерватории почти в два раза.

2) Возможность проводить наблюдения источников космического гамма-излучения, находящихся на небольших угловых расстояниях $\left(1^{\circ}-10^{\circ}\right)$ от Луны [5].

3) Значительно более низкий фон ночного неба, чем в оптическом диапазоне (см., например, [39] и, в контексте работы черенковского телескопа, оценки [7]).

В совокупности с дополнительной возможностью идентификации типа первичной частицы, указанные преимущества позволяют рассматривать использование УФ-чувствительных камер на черенковских телескопах как перспективное направление развития инструментов наземной гамма-астрономии.

Проект гамма-обсерватории ALEGRO предполагает строительство четырех телескопов. Это позволяет рассматривать следующие варианты использования камер с чувствительностью в УФ диапазоне.

1) В темные ночи, когда возможно использование камер с чувствительностью в оптическом диапазоне, УФ камеры могут использоваться на одном или двух телескопах для сравнения сигнала в них с сигналом в оптических камерах других телескопов (с учетом разницы в их местоположении) с целью увеличения надежности определения типа первичной частицы.

2) В лунные ночи, когда работа в оптическом диапазоне невозможна, УФ камеры могут использоваться на всех телескопах обсерватории с целью увеличения длительности рабочего цикла.

При этом для осуществления регистрации в УФ диапазоне могут быть использованы как камеры из фотодетекторов с чувствительностью исключительно в УФ диапазоне (см. например, [6]), так и камеры с фотодетекторами в оптическом и УФ диапазоне, оснащенные фильтрами, блокирующими излучение оптического диапазона (например, [5,11]). Последняя схема обеспечивает значительно более быструю перенастройку камеры (посредством установки фильтра), чем первая, которая требует полной замены камеры оптического диапазона на УФ камеру. Однако такая схема требует создания новых SiPM со значительной чувствительностью как в оптическом, так и в УФ диапазоне. Еще одним возможным вариантом является создание гибридной камеры, в которой УФ и оптические детекторы-пикселы будут расположены в шахматном порядке (что в некотором роде является развитием схемы, использованной в телескопе ГТ-48 [4]), и в случае необходимости будет использоваться маска, закрывающая детекторы оптического диапазона, либо эти детекторы будут отключаться от системы питания.

Выбор конкретной реализации технологии регистрации черенковского излчения ШАЛ в УФ диапазоне будет предметом дальнейших исследований в рамках проекта гамма-обсерватории ALEGRO и других черенковских гамма-телескопов четвертого поколения.

\section{Заключение}

Проведенное моделирование позволяет сделать следующие выводы:

1. Измерение отношения количества УФ фотонов к количеству оптических фотонов ШАЛ, $N_{\mathrm{UV}} / N_{\mathrm{opt}}$, может быть полезным методом повышения надежности гаммаадронной сепарации. Реализация этого метода при эквивалентной энергии первичных частиц $10 \mathrm{GeV}-1 \mathrm{TeV}$ требует измерения величины $N_{\mathrm{UV}} / N_{\mathrm{opt}}$ с точностью $\sim 7 \cdot 10^{-3}$ (т. е. с относительной точностью $\sim 13-8 \%$ ) на высоте $2 \mathrm{~km}$, и с точностью $\sim(1.6-0.7) \cdot 10^{-2}$ (т.е. с относительной точностью $\sim 13-4 \%$ ) на высоте $5 \mathrm{~km}$. Необходимо отметить, что по результатам многочастичного моделирования, которое позволяет принять во внимание флуктуации физических величин, характеризующих ШАЛ, эта оценка может быть ухудшена (т.е. требуемая точность станет выше). Следует ожидать, что при расположении обсерватории на высоте $5 \mathrm{~km}$ метод гамма-адронной сепарации посредством измерения отношения $N_{\mathrm{UV}} / N_{\text {opt }}$ будет более эффективным, чем при расположении на высоте $2 \mathrm{~km}$, поскольку абсолютное количество УФ фотонов на высоте $5 \mathrm{~km}$ примерно в $1.5-2$ раза больше, чем на высоте $2 \mathrm{~km}$ при прочих равных условиях; абсолютная разность величин $N_{\mathrm{UV}} / N_{\text {орt }}$ для ШАЛ от протонов и $\gamma$-квантов с энергией $\lesssim 300 \mathrm{GeV}$ на высоте $5 \mathrm{~km}$ в 1.4-2 раза больше, чем на высоте $2 \mathrm{~km}$.

2. При использовании камер, состоящих исключительно из УФ-чувствительных $\mathrm{SiPM}$, разработанных в ФТИ им. А.Ф. Иоффе, гамма-обсерватория ALEGRO будет иметь пороговую энергию наблюдений $\sim 37 \mathrm{GeV}$ при расположении на высоте $5 \mathrm{~km}$ над уровнем моря, что примерно в два раза ниже, чем при расположении на высоте $2 \mathrm{~km}(\sim 80 \mathrm{GeV})$ при прочих равных условиях.

Полученные результаты могут быть использованы при разработке конструкции и алгоритмов анализа сигналов наземных черенковских гамма-обсерваторий нового поколения.

Е.Е. Холупенко, П.Н. Аруев и А.М. Красильщиков благодарят Российский фонд фундаментальных исследований за поддержку в рамках проекта 16-29-13009-офи-м.

\section{Список литературы}

[1] Gottschall D., FörsterA., Bonardi A., Santangelo A., Puehlhofer $G$. The Mirror Alignment and Control System for CT5 of the H.E.S.S. experiment. In 34th International Cosmic Ray Conference (ICRC2015). Vol. 34 of International Cosmic Ray Conference. P. 1017. July 2015.

[2] Anderhub H., Backes M., Biland A.. Design and operation of FACT - the first G-APD Cherenkov telescope. J. Instrumentation. 2013. Vol. 8. P. 06008. 
[3] Stepanian A.A., Fomin V.P., Vladimirskii B.M. A method of distinguishing Cerenkov gamma-ray flashes from the proton component of cosmic rays. Izvestiya Ordena Trudovogo Krasnogo Znameni Krymskoj Astrofizicheskoj Observatorii. 1983. Vol. 66. P. 234-241.

[4] Zyskin Y.L., Vladimirsky B.M., Neshpor Y.I., Stepanian A.A., Fomin V.P., Shitov V.G. The Investigation of Cerenkov Flashes in Ultraviolet Spectral Region. International Cosmic Ray Conference. 2:342, 1987.

[5] Chantell M., Akerlof C.W., Buckley J., Carter-Lewis D.A., Cawley M.F., Connaughton V, Fegan D.J., Fleury P., Gaidos J., Hillas A.M., Lamb R.C., Pare E., Rose H.J., Rovero A.C., Sarazin X., Sembroski G., Schubnell M.S., Urban M., Wieekes T.C., Wilson C. Gamma-Ray Observations in Moonlight with the Whipple Atmospheric Cherenkov Hybrid Camera. International Cosmic Ray Conference, 2:544, 1995.

[6] Chantell M.C., Sarazin X., Fleury P., Harris K., Kerrick A., Paré E., Punch M., Urban M., Vacanti G., Weekes T.C. The Use of a UV Camera in the Atmospheric Cerenkov Technique. International Cosmic Ray Conference, 2:560, 1995.

[7] Sarazin X., Chantell M.C., Fleury P., Paré E., Punch M., Urban M., Vacanti G., Weekes T.C. // Astroparticle Phys. 1996. Vol. 4. P. 227-234.

[8] Rahman M.A., Bhat P.N., Acharya B.S., Chitnis V.R., Majumdar P., Vishwanath P.R. // Experimental Astronomy. 2001. Vol. 11. P. 113-131.

[9] Rahman M.A., Bhat P.N., Acharya B.S., Chitnis V.R., Majumdar P., Vishwanath P.R. Bulletin of the Astronomical Society of India. 2002. Vol. 30. P. 335-340.

[10] Griffin S. VERITAS Collaboration. VERITAS Observations under Bright Moonlight. In 34th International Cosmic Ray Conference (ICRC2015). Vol. 34 of International Cosmic Ray Conference. P. 989. 2015.

[11] Guberman D., Cortina J., García R., Herrera J., Manganaro M., Moralejo A., Rico J., Will M. MAGIC Collaboration. Using UV-pass filters for bright Moon observations with MAGIC. In 34th International Cosmic Ray Conference (ICRC2015). Vol. 34 of International Cosmic Ray Conference. P. 1237. 2015.

[12] Ahnen M.L., Ansoldi S., Antonelli L.A., Arcaro C., Babić A., and 140 coauthors. // Astroparticle Physics. 2017. Vol. 94. P. $29-41$.

[13] The Cherenkov Telescope Array Consortium: B.S. Acharya, I. Agudo, I.A. Samarai, and 586 coauthors. Science with the Cherenkov Telescope Array. ArXiv e-print 1709.07997, September 2017.

[14] Aharonian F.A., Konopelko A.K., Völk H.J., Quintana H.. // Astroparticle Phys. 2001. Vol. 15. P. 335-356.

[15] Kholupenko E.E., Aruev P.N., Baiko D.A., Bogdanov A.A., Vasilyev G.I., Zabrodskii V.V., Krasilchtchikov A.M., Tuboltsev Y.V., Chichagov Y.V. // Physics of Atomic Nuclei. 2016. Vol. 79. P. $1542-1545$.

[16] Bykov A.M., Aharonian F.A., KrassilchtchikovA.M., Kholupenko E.E., Aruev P.N., Baiko D.N., Bogdanov A.A., Vasilyev G.I., Zabrodskii V.V., Troitsky S.V., Tuboltsev Y.V., Kozhberov A.A., Levenfish K.P., Chichagov Y.V. // J. Techn. Phys. 2017. Vol. 62. P. 819-836.

[17] Zabrodskii V.V., Aruev P.N., Belik V.P., Ber B.Y., Bobashev S.V., Petrenko M.V., SobolevN.A., Filimonov V.V., Shvarts M.Z. // Techn. Phys. Lett. 2014. Vol 40. P. 330-332.

[18] Greisen K. Progress in Cosmic Ray Physics. Vol. 3. Amsterdam: North-Holland, 1956.
[19] Linsley J. GIL: a formula for EAS longitudinal profiles. International Cosmic Ray Conference, 2:502, 2001.

[20] Askaryan G.A. // ZhETF. 1961. Vol. 41. P. 616-618. , 1961. Translated version: Sov. Phys. JETP 14, 441 (1962).

[21] Filonenko A.D., Chekh Y.N. // Russian Radio Physics and Radio Astronomy. 2002. Vol. 7. P. 160.

[22] Giller M., Wieczorek G., Kacperczyk A., Stojek H., Tkaczyk W. // J. Phys. G. Nucl. Phys. 2004. Vol. 30. P. $97-105$.

[23] Ilina N.P., Kalmykov N.N., Prosin V.V. // Sov. J. Nucl. Phys. 1992. Vol.55. P. 1540-1547. [Yad. Fiz. 1992. Vol. 55. P. 2736. ]

[24] Berat C., Bottai S., De Marco D., Moreggia S., Naumov D., Pallavicini M., Pesce R., Petrolini A., Stutz A., Taddei E., Thea A. // Astroparticle Physics. 2010. Vol. 33. P. 221-247.

[25] Ogawa S. Report: First Interaction of Air Shower and Monte Carlo Performance of ASHRA. 1st International Workshop On Air Fluorescence At University of Utah, October 2002.

[26] Heck D., Knapp J., Capdevielle J.N., Schatz G., Thouw T. CORSIKA: a Monte Carlo code to simulate extensive air showers. Forschungszentrum Karlsruhe $\mathrm{GmbH}$, Karlsruhe (Germany)., V + 90 p., TIB Hannover, D-30167 Hannover (Germany), February 1998.

[27] The 1976 Standard Atmosphere Above 86-km Altitude. NASA SP-398. NASA Special Publication. 1976. P. 398.

[28] Bucholtz A. Applied Optics LP. 1995. Vol. 34. P. 2765.

[29] Hamamatsu Photonics. MPPC (multi-pixel photon Counter): S10362-33 series, 2009.

[30] Burrows J.P., Richter A., Dehn A., Deters B., Himmelmann S., Voigt S., Orphal J. // J. Quantitative Spectroscopy and Radiative Transfer. 1999. Vol. 61. P. 509-517.

[31] Orphal J. // J. Photochemistry and Photobiology A: Chemistry. 2003. Vol. 157. P. 185-209.

[32] http:/croc.gsfc.nasa.gov/shadoz; SHADOZ Version: 05; SHADOZ format data created: 31 October, 2011; STATION: Nairobi, Kenya; SHADOZ Principal Investigator: Anne M. Thompson (Penn State University, USA); Station Principal Investigator(s): Bertrand Calpini (MeteoSwiss, Switzerland); Latitude (deg): -1.27; Longitude (deg): +36.80; Elevation (m): 1795.0; Launch Date: 20110126.

[33] Kalekin O.R., Neshpor Y.I., Stepanyan A.A., Zyskin Y.L., Kornienko A.P., Vladimirskii B.M., FominV.P., Shitov V.G.. // Astronomy Letters. 1995. Vol. 21. P. 163-167.

[34] Neshpor Y.I., Chalenko N.N., Stepanian A.A., Kalekin O.R., Jogolev N.A., Fomin V.P., Shitov V.G. // Astronomy Reports. 2001. Vol. 45. P. 249-254.

[35] Zatsepin V.I., Chudakov A.E. // ZhETF. 1962. Vol. 42. P. 1622.

[36] Gaisser T.K., Hillas A.M. Reliability of the method of constant intensity cuts for reconstructing the average development of vertical showers. International Cosmic Ray Conference, 8:353-357. 1977.

[37] Egan A., Fleming B.T., Wiley J., Quijada M., Del Hoyo J., Hennessy J., Hicks B., France K., Kruczek N., Erickson N. The development and characterization of advanced broadband mirror coatings for the far-UV. In Society of Photo-Optical Instrumentation Engineers (SPIE) Conference Series, volume 10397 of Society of Photo-Optical Instrumentation Engineers (SPIE) Conference Series, id. 1039715, August 2017.

[38] Urban M., Chantell M.C., Fleury P., Harris K., Kerrick A.D., Paré E., Punch M., Sarazin X., Vacanti G., Weekes T.C. // Nucl. Instrum. Methods in Phys. Research A. 1996. Vol. 368. P. 503-511.

[39] Leinert C., Bowyer S., Haikala L.K., Hanner M.S., Hauser M.G., Levasseur-Regourd A.C., Mann I., Mattila K., Reach W.T., Schlosser W., Staude H.J., Toller G.N., Weiland J.L., Weinberg J.L., Witt A.N. // Astronomy and Astrophysics Supplement. 1998. Vol. 127. P. 1-99. 